\title{
BMJ Open Ageing stereotypes and prodromal Alzheimer's disease (AGING): study protocol for an ongoing randomised clinical study
}

Kim Gauthier, ${ }^{1}$ Alexandrine Morand ${ }^{2}$ Frederic Dutheil, ${ }^{3,4}$ Béatrice Alescio-Lautier, ${ }^{5}$ José Boucraut, ${ }^{6,7}$ David Clarys, ${ }^{8}$ Francis Eustache, ${ }^{2}$ Nadine Girard, ${ }^{9}$ Eric Guedj, ${ }^{10}$ Marie Mazerolle, ${ }^{11}$ Marc Paccalin, ${ }^{12}$ Vincent de la Sayette, ${ }^{2}$ Aline Zaréa, ${ }^{13}$ Pascal Huguet, ${ }^{3}$ Bernard F Michel, ${ }^{14}$ Béatrice Desgranges, ${ }^{2}$ AGING consortium, Isabelle Régner (i) ${ }^{1}$

To cite: Gauthier K, Morand A, Dutheil F, et al. Ageing stereotypes and prodromal Alzheimer's disease (AGING): study protocol for an ongoing randomised clinical study. BMJ Open 2019:9:e032265. doi:10.1136/ bmjopen-2019-032265

- Prepublication history and additional material for this paper are available online. To view these files, please visit the journal online (http://dx.doi. org/10.1136/bmjopen-2019032265).

$\mathrm{KG}$ and $\mathrm{AM}$ contributed equally.

Received 12 June 2019

Revised 29 August 2019

Accepted 05 September 2019

Check for updates

(C) Author(s) (or their employer(s)) 2019. Re-use permitted under CC BY-NC. No commercial re-use. See rights and permissions. Published by BMJ.

For numbered affiliations see end of article.

Correspondence to Dr Isabelle Régner; isabelle.regner@univ-amu.fr

\section{ABSTRACT}

Introduction The number of older people diagnosed with amnestic mild cognitive impairment (aMCl), the prodromal state of Alzheimer's disease (AD), is increasing worldwide. However, some patients with aMCl never convert to the AD type of dementia, with some remaining stable and others reverting to normal. This overdiagnosis bias has been largely overlooked and gone unexplained. There is ample evidence in the laboratory that negative ageing stereotypes (eg, the culturally shared belief that ageing inescapably causes severe cognitive decline) contribute to the deteriorating cognitive performances of healthy older adults, leading them to perform below their true abilities. The study described here is intended to test for the first time whether such stereotypes also impair patients' cognitive performances during neuropsychological examinations in memory clinics, resulting in overdiagnosis of aMCl.

Methods and analysis The ongoing study is a 4-year randomised clinical trial comparing patients' physiological stress and cognitive performances during neuropsychological testing in memory clinics. A total of 260 patients attending their first cognitive evaluation will be randomised to either a standard condition of test administration, assumed here to implicitly activate negative ageing stereotypes or a reduced-threat instruction condition designed to alleviate the anxiety arising from these stereotypes. Both groups will be tested with the same test battery and stress biomarkers. For 30 patients diagnosed with aMCI in each group $(n=60)$, biomarkers of neurodegeneration and amyloidopathy will be used to distinguish between aMCl with normal versus abnormal $A D$ biomarkers. A 9-month follow-up will be performed on all patients to identify those whose cognitive performances remain stable, deteriorate or improve.

Ethics and dissemination This protocol has been approved by the French National Agency for Medicines and Health Products Safety and the Sud-Est I French Ethics Committee (2017-A00946-47). Results will be published in peer-reviewed journals.

Trial registration number NCT03138018.

\section{Strengths and limitations of this study}

This study represents the first experiment in memory clinics to examine the effects of negative ageing stereotypes on patients' neuropsychological performances.

- It applies a unique multidisciplinary approach to the diagnosis of amnestic mild cognitive impairment (aMCl), combining neurology, neuropsychology, neuroimaging, biology and social psychology.

- This approach will yield a comprehensive picture of the sociopsychological factors liable to bias the diagnosis of aMCl.

- A longer follow-up period may be required than originally anticipated, to analyse conversion and reversion.

- An important next step, not possible with the present design, will be the analysis of how negative ageing stereotypes may prevent efficient activation of the task-relevant brain regions in patients.

\section{INTRODUCTION}

'Few diagnoses in modern medicine evoke deeper apprehension in patient and family than Alzheimer's disease (AD) '. ${ }^{1}$ This fear of developing $\mathrm{AD}$ is quite understandable. It is a progressive and fatal degenerative disorder of the brain that occurs in middle or later life and robs us of our most human qualities and abilities. Its prevalence is generally estimated to be $2 \%$ before age 65 years, $2 \%-4 \%$ thereafter and around $15 \%$ at age 80 years. $^{2}$ Whereas the accuracy of $\mathrm{AD}$ diagnosis in its middle or advanced stage is no longer a real issue, achieving accurate early diagnosis is still a major challenge. ${ }^{3}$ As people are mainly worried about not being diagnosed at an early stage, underdiagnosis is often presented as the main problem. 
However, the opposite error-overdiagnosis-is also likely. Some individuals diagnosed with amnestic mild cognitive impairment (aMCI) never actually convert to $\mathrm{AD}$, with some patients remaining stable and others even reverting to normal ${ }^{4-7}(4.5 \%-53 \%$ reversion to normal rate) ${ }^{8}$ The MCI construct ${ }^{9}{ }^{10}$ designates an early stage of cognitive impairment that is supposed to precede clinically probable $\mathrm{AD},{ }^{11-13}$ and patients with subjective cognitive impairment (SCI) are sometimes considered to be pre-MCI, a stage that can precede $\mathrm{AD}$ by up to 15 years.

The diagnosis of aMCI relies on the neuropsychological testing of memory and cognitive functions to discriminate between normal and abnormal cognitive decline in ageing. It is, thus, essential that these standardised tests and their administration are as fair as possible. Here, we argue that an important source of bias during neuropsychological testing comes from negative ageing stereotypes and the related fear that severe memory decline and $\mathrm{AD}$ are inevitable in older people. These negative ageing stereotypes may exacerbate spontaneous demands by older adults for neuropsychological testing, but also impair their performances on these tests. ${ }^{14-16}$ In the field of social psychology, increasing experimental research conducted in the laboratory among healthy populations has demonstrated that members of groups whose abilities are negatively stereotyped typically underperform when the negative stereotypes are made relevant to the testing situation-a phenomenon called stereotype threat (ST). In addition to the normal anxiety associated with taking tests, the fear of confirming negative stereotypes creates extra pressure, which interferes with intellectual functioning and leads individuals to perform below their abilities. ${ }^{17} 18$

While not denying that normal ageing is associated with cognitive decline, many studies have demonstrated that negative ageing stereotypes contribute to the differences observed in healthy populations between younger and older adults on memory tasks (for reviews, see refs 19-21). ST has been proven to impair older adults' memory performance when differences in abilities between younger and older adults are highlighted, ${ }^{1522} 23$ or when the ageing stereotype is implicitly activated using priming techniques. ${ }^{24-27}$ Likewise, merely informing older adults of the presence of younger participants (without mentioning any expected age-related differences in performance) is enough to decrease their controlled access to memory and intensify their automatic responses. ${ }^{28}{ }^{29}$ ST effects on older adults' memory performances are readily observable and fairly easy to induce with basic instructions of the type typically used in clinics during memory testing. Simply emphasising the memory component of the test can generate performance differences between older and younger adults. ${ }^{3031}$ Interestingly, age-based ST effects can be alleviated and sometimes removed when the memory component of the test is de-emphasised, ${ }^{31}{ }^{32}$ when the test is presented as age fair, ${ }^{29}$ or when older adults are exposed to positive ageing stereotypes. ${ }^{15} 2223$
Several studies have investigated age-based ST effects with classic clinical tests that are typically used for the diagnosis of predementia. ${ }^{33-36}$ Mazerolle et $a l^{36}$ found that ST impaired older adults' performances on both the Mini-Mental State Examination $\left(\mathrm{MMSE}^{37}\right.$ and the Montreal Cognitive Assessment, ${ }^{38}$ resulting in $40 \%$ of older adults meeting the criteria for predementia, compared with $10 \%$ in a reduced-ST condition. Using a comprehensive test battery comparable to those used in clinical settings, Fresson $e t a b^{34}$ found that performances on executive cognitive tasks were impaired in an ST condition, leading to $28 \%$ of older adults meeting the clinical criteria, compared with $8 \%$ in a reduced-ST condition. A recent meta-analysis ${ }^{21}$ revealed that age-based ST effects are robust, with significant effect sizes for older adults' memory ( $\mathrm{d}=0.21,95 \%$ CI .020 to .385$)$ and cognitive performance ( $\mathrm{d}=0.68,95 \%$ CI .399 to .845$)$ that can be even larger when the threat is more subtly induced by the situation ( $\mathrm{d}=0.52,95 \%$ CI .248 to .717$)$. Nonetheless, these were experimental laboratory studies conducted among healthy older adults and relying on ST and/ or reduced-ST instructions that do not correspond to the clinical context in which older adults with cognitive complaints seek a diagnosis. The present study is, therefore, the first to test age-based ST effects among patients in a clinical setting and during neuropsychological testing for aMCI. It is based on the assumption that negative ageing stereotypes implicitly permeate neuropsychological testing in memory clinics and thus impair older adults' performance, potentially resulting in the overdiagnosis of aMCI.

\section{METHODS}

\section{Aim and objectives}

AGING is a multicentre two-armed randomised controlled trial designed to compare older adults' performances on a comprehensive neuropsychological test battery (the same for all patients) under either a standard (ST condition) or reduced-ST condition. The standard condition replicates the one in which testing is routinely conducted in memory clinics. It tests the assumption that routine tests administration implicitly activates negative ageing stereotypes and the fear of having $\mathrm{AD}$. If this assumption is correct, cognitive performances will be poorer in this standard condition than in a condition where ST is reduced via so-called teaching instructions, where a video gives participants an explanation of ST that has been shown to efficiently alleviate anxiety arising from negative stereotypes. $^{36} 39$

\section{Primary objective}

The study's primary objective is to test whether fewer patients meet the aMCI criteria in the reduced-ST condition than in the standard condition, by comparing their cognitive performances. For some of those diagnosed with aMCI in each condition, biomarkers of neurodegeneration (MRI hippocampal volume, perfusion positron 
emission tomography (PET) imaging, cerebrospinal fluid (CSF) levels of $\beta$-amyloid, total-tau and phospho-tau) and amyloidopathy (PET imaging) will be used to improve diagnostic accuracy, by distinguishing between aMCI with abnormal $\mathrm{AD}$ biomarkers (ie, aMCI due to $\mathrm{AD}$ ) and $\mathrm{aMCI}$ with normal $\mathrm{AD}$ biomarkers.

\section{Secondary objectives}

1. Examine, through the use of biomarkers of stress (heart rate variability (HRV), skin conductance and salivary biomarkers), the extent to which age-based ST induces acute physiological stress during the neuropsychological assessment.

2. Conduct a 9-month follow-up to identify participants whose cognitive performances remain stable, deteriorate or improve.

3. Administer a questionnaire, to pinpoint the individual characteristics (vulnerability factors) that can make older people more or less susceptible to ST bias during neuropsychological testing (eg, stereotypical perceptions of ageing, memory complaints, anxiety about ageing and subjective age).

4. Develop an efficient method for deactivating the influence of negative ageing stereotypes during neuropsychological testing and provide new recommendations to improve the diagnosis accuracy of aMCI due to $\mathrm{AD}$.

5. Study the brain substrate of memory evaluation under ST versus reduced-ST conditions using MR volumetry and PET perfusion.

\section{Procedure overview}

This protocol follows the Standard Protocol Items: Recommendations for Interventional Trials (SPIRIT) guidelines (see SPIRIT checklist in online supplementary file) ${ }^{40}$ Over a period lasting from July 2018 to December
2020, the aim is to recruit 260 participants among individuals with memory complaints but without probable $\mathrm{AD}$ attending a memory clinic for their first cognitive assessment. Figure 1 indicates the four visits planned in the study. Consistent with the traditional clinical procedure, the first visit consists of a brief screening test conducted by a neurologist, who decide on this basis whether or not to pursue more in-depth cognitive testing. Patients selected for this in-depth testing are invited to take part in the study and receive the consent form and a questionnaire (ST vulnerability factors) to complete at home and bring on their next visit. During this second visit, within 2 months of the screening, a neuropsychologist administers a comprehensive neuropsychological test batterythe same for all of patients-under either standard or reduced-ST instructions (random assignment based on a computer-generated allocation sequence and controlled by the central site). In addition to cognitive performances, several biomarkers of stress are measured. The neuropsychological assessment leads to a diagnosis of SCI, aMCI or possibly AD. A proportion of the patients diagnosed with aMCI ( $\mathrm{n}=60 ; 30$ from each arm) are assigned to a third visit, during which biomarkers of neurodegeneration and amyloidopathy (MRI hippocampal volume, $\beta$-amyloid tracer) are used to distinguish between aMCI with abnormal versus normal AD biomarkers. Finally, at 9-month follow-up (fourth visit), all patients are tested in a reduced-ST condition (whatever their previous testing condition) with the same neuropsychological battery as before and physiological stress measures to identify patients whose cognitive performances have remained stable, worsened or improved. The neuropsychology testing is performed in four centres in France: Marseille Public Hospitals (central site), La Milétrie University

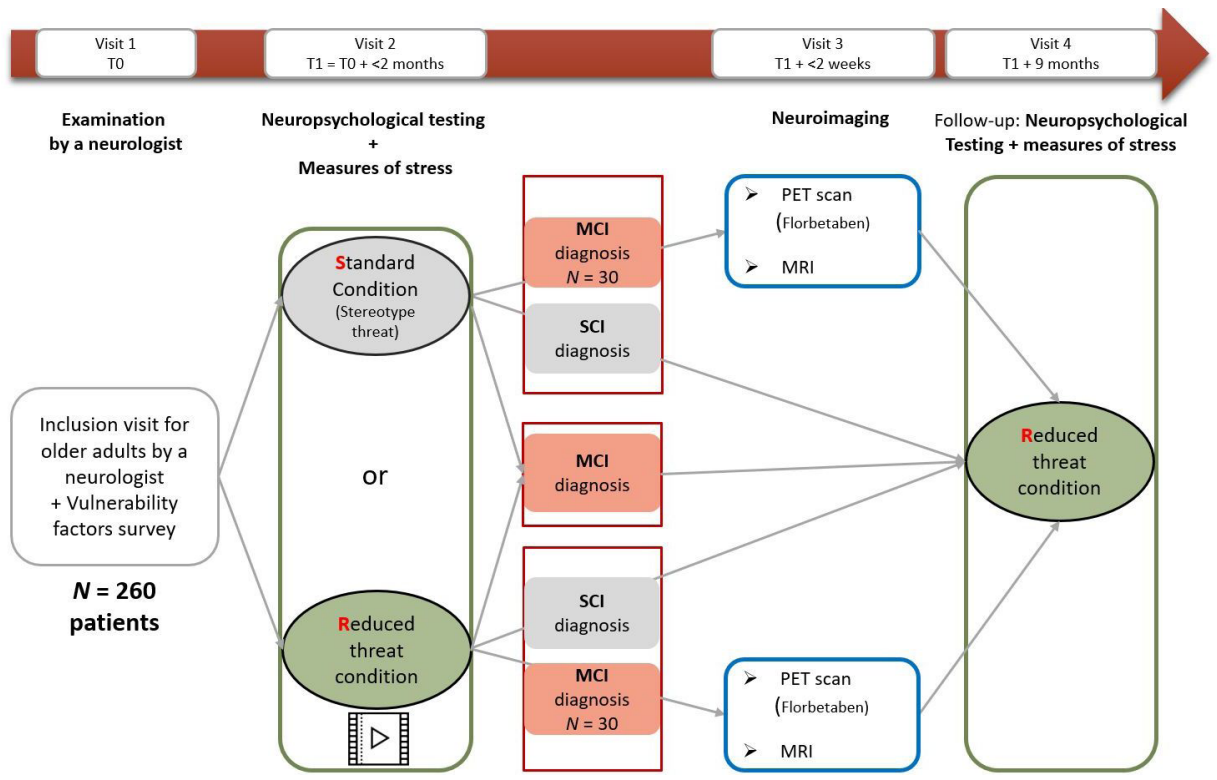

Figure 1 Flow chart of the main steps for patients. As a routine procedure, aMCl patients will be offered a lumbar puncture (included in the consent form). aMCl, amnestic mild cognitive impairment; $\mathrm{MCl}$, mild cognitive impairment; PET, positron emission tomography; SCl, subjective cognitive impairment. 
Hospital in Poitiers, Caen University Hospital and Charles Nicolle University Hospital in Rouen. The neuroimaging explorations (MRI, PET) are performed in Marseille (Hospital) and Caen (Cyceron neuroimaging centre). Risks to participating patients are minimal and linked mainly to the neuroimaging part of the research. Study staff in each hospital complete case report forms during each visit and notify the principal investigator and clinical investigators of any adverse events.

\section{Inclusion and non-inclusion criteria}

Inclusion in the cohort is offered to patients by a neurologist during the first medical examination (visit 1), based on their background and their responses to the following short cognitive tests: Questionnaire de Plainte Cognitive $(\mathrm{QPC}){ }^{41}$ Geriatric Depression Scale (GDS ${ }^{42}$ MMSE, ${ }^{37}$ 5-word test ${ }^{43}$ and Lawton Instrumental Activities of Daily Living (IADL) Scale. ${ }^{44}$ The patients targeted by the study are SCI (cognitive complaints without cognitive decline) and/or MCI patients (cognitive complaints with cognitive decline but without $\mathrm{AD}$ dementia). To be included, patients must be at least 50 years old, report cognitive complaints and meet the following criteria: QPC score $\geq 3$, GDS score $\leq 9$, MMSE score $\geq 24$, 5 -word test score $>8$ and IADL score $=0$. Patients presenting signs of probable $\mathrm{AD}$ according to the NINCDS-ADRDA (National Institute of Neurological and Communicative Diseases and Stroke/Alzheimer's Disease and Related Disorders Association) criteria are not enrolled in AGING. Other non-inclusion criteria include prior or current traumatic brain injury, neurological or cardiovascular disorders, psychiatric disorder (schizophrenia, bipolar disorder and major depression), use of psychotropic medication and alcohol abuse.

\section{Study arms}

At the beginning of visit 2, before taking the neuropsychological test battery, patients are randomly assigned to one of two arms: standard test instructions or reduced-ST instructions. The randomisation is computer generated, based on an Excel file (created by an independent coworker), and is performed using permuted blocks $($ size $=4)$. Within each hospital, randomisation is performed according to the patients' age, sex and socioeconomic status, in order to ensure that the groups are balanced in terms of size and patient characteristics that are of potential importance for investigating susceptibility to age-based ST effects. Patients, neuroimaging staff and neurologists are all blinded to the conditions, except for the neuropsychologists, who need to know which instructions to use. To ensure the blinding of the diagnosis, neuropsychologists who were in charge of the neuropsychological evaluation will not participate in the diagnosis decision-making.

The standard test instructions replicate the instructions traditionally used in memory clinics during neuropsychological assessments. These instructions have simply been standardised across the four centres. Patients are informed by the neuropsychologist that they are about to undergo a more in-depth assessment than they did during the consultation with the neurologist, in order to test their memory, language, and all the functions they use in daily life. The neuropsychologist emphasises that the testing includes tasks of varying difficulty, so it is normal not to be successful on all the tests. Finally, all the patients are encouraged to do their very best and the testing process then begins. While one might intuitively consider this standard condition to be a neutral condition that does not activate ST, we assume that it implicitly promotes ST effects and is self-threatening for patients.

The reduced-ST instructions are designed to reduce ST by using so-called teaching instructions, which consist in explaining ST to patients in order to alleviate any anxiety arising from negative ageing stereotypes. In order to standardise and optimise the effectiveness of these instructions, we have developed a 4 min video showing a female or male patient during an interview in a memory clinic. For identification purposes, the gender of the fictitious patient is matched to that of each participant. The video shows a patient coming to a memory clinic for an initial neuropsychological assessment because of memory concerns. Several scenes are enacted and represent the usual steps in the circuit of an MCI/AD diagnosis. The patient meets a neurologist first, then a neuropsychologist, who both give the following teaching instructions to reduce ST: 'In our societies, we are all exposed in the media to sensational and often fearful information about $\mathrm{AD}$ that inevitably induces a lot of stress. This stress can lead people to experience difficulties during the testing, which can impair performances. The tests themselves can be stressful. It is, thus, normal to make some mistakes due to stress, but these mistakes are not necessarily signs of AD'. We chose not to use a video in the standard (control) condition, in order to stay as close as possible to classic testing practices during neuropsychological assessments in memory clinics.

\section{MEASURES}

\section{Sample size}

The number of participants needed in the study was determined $^{45}$ on the basis of the anticipated effect size of the ST. Lamont et $a l^{21}$ conducted a meta-analysis of age-based ST and found an effect size of $\mathrm{d}=0.52$ (95\% CI .248 to .717 , corresponding to $\left.f^{2}=0.15\right)$, when they manipulated ST as we do here. However, as ours is the first study of age-based ST in a clinical setting, we decided to use a lower effect size $\left(\mathrm{f}^{2}=0.07\right)$ to determine our target sample size, in order to have sufficient power to accurately detect a probably smaller effect in an ecologically valid field experiment. Using this smaller effect size, with the error rate set at 0.05 , and power set at 0.80 , a power analysis ${ }^{46}$ indicated that a sample of 250 participants would be sufficient to detect the critical effects of the conditions and their potential interactions with several moderators in a multiple regression analysis (11 predictors: condition, age, physiological stress, 4 vulnerability factors and the 
Table 1 Neuropsychological test battery used in the study for visits 2 and 4

\begin{tabular}{|c|c|c|c|c|}
\hline & Test & $\begin{array}{l}\text { Duration } \\
\text { (min) }\end{array}$ & Domain assessed & Authors \\
\hline 1 & $\begin{array}{l}\text { 16-item Free and Cued Selective Reminding Test, with } \\
\text { parallel list for 9-month follow-up (Visit 4) }\end{array}$ & 20 & Episodic memory & Van der Linden et $a l^{52}$ \\
\hline 2 & Visuospatial focused attention task & 5 & $\begin{array}{l}\text { Visuospatial } \\
\text { attentional flexibility }\end{array}$ & Herrera et al ${ }^{62}$ \\
\hline 3 & Parts $A$ and $B$ of the Trail Making Test & 5 & Flexibility & Reitan 63 \\
\hline 5 & N-back task & 5 & Working memory & $\begin{array}{l}\text { Adapted from Perlstein } \\
\text { et } a l^{65}\end{array}$ \\
\hline 6 & Category and lexical fluency task & 5 & $\begin{array}{l}\text { Verbal fluency } \\
\text { (language) }\end{array}$ & Cardebat et a/ ${ }^{66}$ \\
\hline 7 & $\begin{array}{l}\text { Rey-Osterrieth Complex Figure (copy and delayed } 3 \text { min } \\
\text { recall) for visit 2, and Taylor figure for visit } 4\end{array}$ & $5-7$ & $\begin{array}{l}\text { Visuoconstruction and } \\
\text { memory }\end{array}$ & Osterrieth, Rey, Taylor ${ }^{67-69}$ \\
\hline 9 & MemPro test & 15 & Prospective memory & $\begin{array}{l}\text { Adapted from Gonneaud } \\
\text { et } a l^{71}\end{array}$ \\
\hline
\end{tabular}

interaction terms between condition and these vulnerability factors). We added 10 participants as a safety margin $(n=260)$, in order to ensure that at least 30 participants in each arm meet aMCI criteria for visit 3. Patients will be continuously recruited until the desired sample size is achieved, but not beyond December 2020, to allow sufficient time for the 9-month follow-up before September 2021. Since February 2019, strategies have been implemented to increase recruitment: communication on the AGING protocol has been improved (eg, poster displays in hospitals and general practices, call for participation in AGING on hospital websites) and appointment scheduling and reminders via post, email and/or telephone are being used to retain patients already enrolled in the trial.

\section{Vulnerability factors to ST effects}

In addition to demographic information (sex, date of birth, years of education, socioeconomic status, native language, etc), a questionnaire captures participants' reports of memory complaints Illness Perception Questionnaire-Memory ${ }^{47}$ their stereotypical perceptions of ageing and $\mathrm{AD},{ }^{26}{ }^{48}$ and their self-categorisation as older versus younger. ${ }^{36}$ Participants complete this questionnaire at home and bring it back to the memory clinic at visit 2 . These variables will be tested as potential moderators of ST effects on cognitive and stress outcomes.

\section{Neuropsychological test battery (visits 2 and 3 )}

The same neuropsychological test battery (table 1) is used for all patients, whatever the condition to which they are randomly assigned (standard vs reduced-ST condition). All the tests are commonly used in memory clinics, except for the second one assessing visuospatial attentional flexibility and the last one assessing prospective memory.
To be categorised as having aMCI or non-aMCI (naMCI), participants have to meet the corresponding criteria of the revised NINCDS-ADRDA standards. ${ }^{450}$ Our MCI categorisation is based on the Free and Cued Selective Reminding Test (FCSRT) (either total recall below or equal to $40 / 48$, Sarazin et $a l^{51}$ or scores below Van der Linden's pathological thresholds. ${ }^{52}$ The $z$-scores obtained for the other cognitive function tests are used to complete the diagnosis, conduct between-group comparisons (standard vs reduced-ST conditions), and perform within-participants comparisons based on the 9-month follow-up. These additional tests enable us to distinguish between aMCI affecting single versus multiple domains. For the patients with naMCI (if any), the diagnosis will be established at the end of the study. The raw scores are used for the tests without normative data (ie, visuospatial focused attention and MemPro). Participants who report memory complaints but have normal cognitive performances are categorised as having SCI.

\section{Physiological stress measures}

We use HRV, skin conductance and biomarkers of stress to measure physiological stress (table 2) during the neuropsychological testing at visit 2 under either standard or reduced-ST conditions, and during the follow-up visit. These three measures of physiological stress will be tested as potential mediators of the ST effect on cognitive performances and as complementary indicators of any potential progression of the disease.

\section{Analysis of HRV}

HRV data will first be examined according to Task Force recommendations. ${ }^{53}$ Premature atrial and ventricular beats and the subsequent intervals will be automatically 
Table 2 Characteristics of devices used to measure physiological stress

\begin{tabular}{|c|c|c|}
\hline Measure & Device & Characteristics \\
\hline Heart rate variability & $\begin{array}{l}\text { Thin elasticated heart rate transmitter belt } \\
\text { placed around the chest }\end{array}$ & $\begin{array}{l}\text { Can detect heart rates of } 25-240 \text { beats per minute } \\
\text { and respiratory rates of } 3-70 \text { breaths per minute }\end{array}$ \\
\hline Stress biomarkers & $\begin{array}{l}\text { Salivette. A synthetic cotton swab is removed } \\
\text { from the Salivette and placed in the mouth of } \\
\text { the participant to chew for about } 1 \mathrm{~min}\end{array}$ & $\begin{array}{l}\text { The Salivette is stored at }-80^{\circ} \mathrm{C} \text { and then sent to a } \\
\text { laboratory for analysis }\end{array}$ \\
\hline
\end{tabular}

discarded and visually checked. We will simultaneously explore HR data in time and frequency domains. In the time domain, we will analyse R-R intervals, the SD of R-R intervals, the square root of the mean squared difference of successive R-R intervals (rMSSD) and the number of adjacent N-N differing by more than 50 milliseconds, divided by the total number of N-N intervals (pNN50). The rMSSD and pNN50 are associated with high-frequency power (HF) and parasympathetic activity. In the spectral domain, we will analyse low-frequency power (LF; $0.04-0.15 \mathrm{~Hz}$ ), an index of both sympathetic and parasympathetic activity, and HF $(0.15-0.4 \mathrm{~Hz})$, representing the most efferent vagal (parasympathetic) activity to the sinus node. The LF/HF ratio (ie, sympathovagal balance) will also be calculated.

\section{Analysis of stress biomarkers}

Biomarkers of stress include cortisol, dehydroepiandrosterone and its sulphated stable form from the HPA axis, and IgA. We perform assays of saliva samples taken at two time points (on arrival at the clinic and before leaving). For biomarkers of stress (cortisol, etc), sensitivity and intra-assay and interassay coefficients of variation are below $0.05 \mathrm{ng} / \mathrm{mL}, 8 \%$ and $10 \%$, for all biomarkers. ${ }^{54}$

\section{Neurodegeneration and amyloidopathy biomarkers}

For the patients diagnosed with aMCI after the neuropsychological examination (30 in the standard condition and 30 in the reduced-ST condition), neuroimaging biomarkers are used to identify those with a high likelihood of $\mathrm{AD}$ aetiology: those showing positive $\mathrm{AD}$ biomarkers of both neuronal injury (MRI: hippocampal atrophy) and $\beta$-amyloid deposition (florbetaben PET), as recommended by Dubois et al..$^{55}$ Early florbetaben PET acquisitions are conducted to evaluate brain perfusion. ${ }^{56}$ The hospitals involved in the present study routinely use the CSF biomarkers $\beta$-amyloid and tau, owing to their high diagnostic accuracy in detecting early or even prodromal AD. ${ }^{4}$ A lumbar puncture is, therefore, offered as a routine procedure to all patients categorised as having aMCI (ie, at risk of $\mathrm{AD}$ conversion) on the basis of their neuropsychological performances at visit 2. It is notified in the consent form that some patients will be asked for their consent to use their CSF data within the AGING protocol (see in online supplementary file, appendix I).

\section{Analysis of MRI data}

Half the patients will undergo an MRI session at the Cyceron centre (Caen, France) with a Philips (Eindhoven, The Netherlands) Achieva 3.0 T scanner. A high-resolution T1-weighted anatomical image is acquired using a three-dimensional (3D) fast field echo sequence (sagittal; $20 \mathrm{~ms}$ repetition time; $4.6 \mathrm{~ms}$ echo time; $10^{\circ}$ flip angle; 180 slices; $1 \mathrm{~mm}$ slice thickness; $256 \times 256 \mathrm{~mm} 2$ field of view; $256 \times 256$ matrix). The other half will undergo an MRI session at the imaging centre in Marseille with a Siemens (Erlangen, Germany) MAGNETOM Skyra syngox MR D13 3T scanner. The high-resolutionT1-weighted images are obtained with the 3D magnetisation prepared rapid gradient echo sequence (sagittal; $2300 \mathrm{~ms}$ repetition time; $2.98 \mathrm{~ms}$ echo time; $9^{\circ}$ flip angle; $900 \mathrm{~ms}$ inversion time; 176 slices; $1 \mathrm{~mm}$ slice thickness; $256 \times 256 \mathrm{~mm}^{2}$ field of view; $256 \times 256$ matrix). In both centres, participants are given earplugs and their head is stabilised with foam pads to minimise head motion.

Visual analyses will be independently performed by two experts who will be blinded to the clinical data and the experimental conditions. In the case of discrepancies between the two experts, an analysis will be performed by a third expert and the final result will be reached by consensus. Particular attention will be paid to the amygdala-hippocampal complex as a marker of neurodegeneration. A whole-brain voxel-based analysis will be also conducted on SPM12 (https://www.fil.ion.ucl.ac.uk/ $\mathrm{spm} /$ software/spm12/) after cortical segmentation, spatial normalisation and smoothing, to study correlations between memory performances in the standard versus reduced-ST condition and grey-matter density.

\section{Analysis of PET data: amyloid load (florbetaben 18F)}

Amyloid load is measured using florbetaben (18F) (NEURACEQ; Piramal Imaging, Cambridge, UK), a radiotracer with high sensitivity for $\beta$-amyloid deposition and a physical half-life (110 $\mathrm{min})$ that makes it suitable for our laboratory setup. Participants are examined using whole-body PET/CT scanners: Dual-Gemini (Philips Medical Systems) in Caen and Discovery 710 (General Electric) in Marseille. A low-dose CT scan is performed first $(600 \mathrm{~mm}$ scanning range, $5 \mathrm{~mm}$ increment, $3.2 \mathrm{~mm}$ cutting thickness; 75 s per rotation, $512 \times 512$ matrix, 120 $\mathrm{kV}, 80 \mathrm{mAs}$ ), followed by a brain scan lasting 20 min that 
starts 90 min after injection of a dose of $300 \mathrm{MBq}$ of florbetaben (18F) (minimum dose $240 \mathrm{MBq}$, maximum dose $360 \mathrm{MBq}$, recommended dose $300 \mathrm{MBq}$ ). An additional 5 min acquisition takes place 1 min after injection to assess brain perfusion.

Visual analyses will first be independently performed by two experts blinded to the clinical data and experimental conditions. In the case of discrepancies between the two experts, an analysis will be performed by a third expert and the final result will be reached by consensus. In order to validate the results of the visual analyses, the molecular imaging experts will assess the standard uptake value ratio (SUVr), ${ }^{57}$ a semiquantitative index. An SUV will be obtained for each region of interest (ROI). As the cerebellum is reported to be free of fibrillar amyloid plaques in the $\mathrm{AD}$ brain, we will use the region-to-cerebellum SUVrs for the between-participants comparison, considering both the whole brain and ROIs. Each regional SUVr value will be expressed as the mean over the ROI. To evaluate florbetaben $(18 \mathrm{~F})$ uptake, we will use 13 ROIs, with values averaged for each one (precuneus, anterior cingulate, posterior cingulate, frontal, temporal, parietal, occipital, hippocampus, centrum semiovale, anterior putamen, posterior putamen, caudate nucleus and pons, as defined in the MNI-AAL atlas). We will use neocortical SUVr florbetaben (18F) values to distinguish amyloid-positive patients from amyloid-negative patients, using a threshold florbetaben (18F) SUVr value of between 1.4 and 1.5, in agreement with previous studies using florbetaben $(18 \mathrm{~F}) .{ }^{585}$ We will reconstruct all PET sinograms with a 3D iterative algorithm, with corrections for randomness, dispersion, photonic attenuation and decomposition, imaging with an isotropic voxel of $2 \times 2 \times 2 \mathrm{~mm}^{3}$ and a spatial resolution of approximately 2-3 mm wide at mid-height of the central field of view. A whole-brain voxel-based analysis will be also conducted on SPM12 (https://www.fil.ion.ucl.ac.uk/spm/software/ spm12/) after spatial normalisation and smoothing, to study correlations between memory performances in the standard or reduced-ST condition and brain perfusion and $\mathrm{A} \beta$ burden.

\section{Analysis of CSF data}

CSF data collected in routine practice will be integrated into AGING data.

The CSF-based diagnosis of aMCI due to $\mathrm{AD}$ relies on a combination of lowered $\beta$-amyloid peptide (1-42 and 1-40) and increased tau and phospho-tau protein curves.

\section{Statistical analyses}

Analyses will be conducted in a blinded way, with codes assigned to conditions (A vs B) and diagnostic categories (numbers). The characteristics of all the demographic, clinical and biological data that are collected, both at baseline and at each visit, will be reported using descriptive statistics. The continuous data will be summarised using mean, SD, median and range values, and the normality assumption will be systematically checked. The categorical data will be presented in frequency tables (n, \%). Missing data will be handled according to the missing at random hypothesis (eg, multiple imputation, longitudinal mixed-effects models), as recommended for missing data in clinical trials involving patients with potential neurodegenerative disease. ${ }^{60}$

To achieve the primary objective (testing whether fewer patients meet the aMCI criteria in a reduced-ST condition than in the standard condition), analyses will first be conducted on the whole sample, using multiple linear regression on the FCSRT scores and logistic regression analysis on the diagnostic categories, with both analyses controlling for demographic variables. These analyses will then be rerun on the subsample of 60 patients with a potentially refined diagnosis based on the combination of neurodegeneration and amyloidopathy biomarker data.

To achieve the secondary objectives, we will run a mediation analysis to estimate whether the standard (threatening) condition induces acute physiological stress during the neuropsychological assessment (compared with the reduced-ST condition) that impairs cognitive performances. Mixed models will be performed to compare patients' cognitive performances on the neuropsychological assessment between visits 2 and 4 , in order to identify patients whose scores remain stable, deteriorate or improve. Finally, moderation and conditional process analyses will be performed on cognitive performances to identify whether some individual characteristics (vulnerability factors: stereotypical perceptions of ageing, memory complaints, anxiety about ageing, subjective age) can moderate patients' susceptibility to age-based ST effects.

\section{Data management and monitoring}

Study staff are responsible for data entry and range checks for data values. All data are stored on a secure server provided by one of the universities. Given the minimal risk nature of the study, the data are internally monitored (Marseille Public Hospitals, which is independent of the funder). Reports on study progress and milestones are submitted to the funder each year.

\section{Patient and public involvement}

Patients and members of the public were not involved in the development of this study protocol. However, alleviating the anxiety that patients can experience due to negative ageing stereotypes during neuropsychology testing and thereby helping to improve the accuracy of MCI diagnosis was a major motivation for the AGING consortium to develop and conduct this study. The findings will, therefore, be disseminated to participants and to the community at large through newsletters and conferences.

\section{Ethics and dissemination}

Written informed consent is required from each patient prior to inclusion. Those people who have direct access to the data take all necessary precautions to maintain 
confidentiality. All data collected during the study are rendered anonymous. Only the inclusion number is registered.

The results will be disseminated via peer-reviewed publications, conferences, and clinical networks targeting researchers, policy- makers, clinicians and caregivers.

\section{CONCLUSION}

$\mathrm{AD}$ is a progressive disease that results in dependence and a need for institutionalisation, and is so far untreatable. Ethical questions are raised by the overdiagnosis of aMCI, as its consequences can be extremely damaging for the patient and for the family and society. ${ }^{61}$ Without denying that ageing is associated with cognitive decline and neurodegenerative diseases for many people, we suggest that special attention should be paid to the influence of psychosocial factors that have been largely overlooked, regarding neuropsychological testing in memory clinics. The AGING project has potentially important theoretical and practical implications for improving neuropsychological testing, to avoid many older people from being wrongly classified as having aMCI.

\section{Trial status}

The recruitment phase began in July 2018 for Marseille Public Hospitals, September 2018 for Poitiers University Hospital, October 2018 for Caen University Hospital and November 2018 for Rouen University Hospital. Twenty-eight patients had been recruited at the time of the study. The estimated end date for this study is September 2021.

\section{Author affiliations}

${ }^{1}$ Aix Marseille Univ, CNRS, LPC, Marseille, France

${ }^{2}$ Normandie Université, UNICAEN, PSL Universités Paris, EPHE, INSERM, U1077, CHU de Caen, Neuropsychologie et Imagerie de la Mémoire Humaine, Caen, France ${ }^{3}$ Université Clermont Auvergne, CNRS, LAPSCO, Clermont-Ferrand, France

${ }^{4}$ Physiological and Psychosocial Stress, University Hospital of Clermont-Ferrand, CHU Clermont-Ferrand, Preventive and Occupational Medicine, WittyFit, ClermontFerrand, France

${ }^{5}$ Aix Marseille Univ, CNRS, LNSC, Marseille, France

${ }^{6}$ Immunology Laboratory, Assistance Publique-Hôpitaux de Marseille, Conception Hospital, Marseille, France

${ }^{7}$ Timone Neuroscience Institute, Aix-Marseille Univ, Marseille, France

${ }^{8}$ Centre de Recherches sur la Cognition et I'Apprentissage, CNRS, Université de Poitiers, Université de Tours, Poitiers, France

${ }^{9}$ Aix Marseille Univ, CRMBM UMR CNRS 7339, APHM Timone Neuroradiologie, Marseille, France

${ }^{10}$ Aix Marseille Univ, CNRS, Ecole Centrale Marseille, UMR 7249, Institut Fresnel, \& Department of Nuclear Medicine, Assistance Publique-Hôpitaux de Marseille, Timone University Hospital, Marseille, France

${ }^{11}$ Department and Laboratory of Psychology, MSHE, Université Bourgogne FrancheComté, Besançon, France

${ }^{12}$ Centre d'Investigation Clinique CIC 1402, INSERM, Centre Hospitalier Universitaire de Poitiers, Poitiers, France

${ }^{13}$ Department of Neurology, Rouen University Hospital and University of Rouen, Rouen, France

${ }^{14}$ Departement of Neurological Behavior, Assistance Publique-Hôpitaux de Marseille, Sainte-Marguerite University Hospital, Marseille, France

Collaborators Members of AGING Consortium: Hospitals: José Broucraut, Boris Dufournet, Carine Giallo, Nadine Girard, Eric Guedj, Bernard François Michel,
Nathalie Sambuchi (Marseille Public Hospitals, France: central site); Pierre Branger, Julien Cogez, Sabine Fradin, Frédérique Grandhomme, Catherine Lalevée, Nadège Loisel, Olivier Martinaud, Alice Pélerin, Mélissa Pierre, Vincent de La Sayette (Caen University Hospital, France); Alain Manrique (Cyceron Biomedical Imaging Centre, Caen, France); Vanessa Baudifier, Foucaud du Boisgueheneuc, Adrien Julian, JeanClaude Lecron, Isabelle Merlet, Claudie Ornon, Marc Paccalin (Poitiers University Hospital, France); Sandrine Bioux, Evangeline Bliaux, Didier Hannequin, Véronique Hannier, Maud Loubeyre, Dorothée Pouliquen, Muriel Quillard-Muraine, David Wallon, Aline Zaréa (Rouen University Hospital, France).Laboratories: Kim Gauthier, Isabelle Régner (Cognitive Psychology Laboratory, Aix Marseille Univ, Marseille, France); Béatrice Alescio-Lautier, Véronique Paban, Arnaud Weill (Sensory and Cognitive Neuroscience Laboratory, Aix Marseille Univ - CNRS, Marseille, France); David Clarys, Michel Isingrini, Sandrine Kalenzaga, François Rigalleau (Centre for Research on Cognition and Learning, CNRS - Poitiers University - Tours University, Poitiers, France); Ahmed Abbas, Julien Chavant, Béatrice Desgranges, Francis Eustache, Florence Fraisse, Mickael Laisney, Alexandrine Morand, Shailendra Segobin (Neuropsychology and Human Memory Imaging research unit, Caen University Hospital - Caen Normandy University - PSL Research University - EPHE - INSERM, Caen, France); Pierre Chausse, Frédéric Dutheil, Julien Guegan, Pascal Huguet, Guillaume Vallet (Social and Cognitive Psychology Laboratory, University of Clermont Auvergne - CNRS, Clermont-Ferrand, France); Sophie Blanchet, Pascale Piolino (Memory and Cognition, Institute of Psychology and Centre for Psychiatriy and Neurosciences, INSERM - University of Paris Descartes, Paris, France); Marie Mazerolle (Psychology Department and Laboratory, University of Bourgundy Franche-Comté, France).

Funding This study was supported by a grant from the French National Research Agency (ANR) (grant no. ANR-16-CE36-0005-01).

Competing interests None declared.

Patient consent for publication Not required.

Ethics approval This protocol was originally approved in July 2017 and the present study details the protocol contained in the latest version approved in April 2019. All changes to the study were decided by the consortium and reviewed by the French National Agency for Medicines and Health Products Safety and the Sud-Est I French Ethics Committee.

Provenance and peer review Not commissioned; externally peer reviewed.

Open access This is an open access article distributed in accordance with the Creative Commons Attribution Non Commercial (CC BY-NC 4.0) license, which permits others to distribute, remix, adapt, build upon this work non-commercially, and license their derivative works on different terms, provided the original work is properly cited, appropriate credit is given, any changes made indicated, and the use is non-commercial. See: http://creativecommons.org/licenses/by-nc/4.0/.

ORCID iD

Isabelle Régner http://orcid.org/0000-0002-2256-139X

\section{REFERENCES}

1 Selkoe DJ. Preventing Alzheimer's disease. Science 2012;337:1488-92.

2 Blum D, Buée L, INSERM. Alzheimer (maladie d'): une maladie neurodégénérative complexe mais de mieux en mieux comprise, 2019. Available: https://www.inserm.fr/information-en-sante/ dossiers-information/alzheimer-maladie

3 Tian T, Zhang B, Jia Y, et al. Promise and challenge: the lens model as a biomarker for early diagnosis of Alzheimer's disease. Dis Markers 2014;2014:1-5.

4 Ewers M, Walsh C, Trojanowski JQ, et al. Prediction of conversion from mild cognitive impairment to Alzheimer's disease dementia based upon biomarkers and neuropsychological test performance. Neurobiol Aging 2012;33:1203-14.

5 Han JW, Kim TH, Lee SB, et al. Predictive validity and diagnostic stability of mild cognitive impairment subtypes. Alzheimers Dement 2012;8:553-9.

6 Morris JC, Storandt M, Miller JP, et al. Mild cognitive impairment represents early-stage Alzheimer disease. Arch Neurol 2001;58:397-405.

7 Solfrizzi V, Panza F, Colacicco AM, et al. Vascular risk factors, incidence of $\mathrm{MCl}$, and rates of progression to dementia. Neurology 2004;63:1882-91.

8 Sachdev PS, Lipnicki DM, Crawford J, et al. Factors predicting reversion from mild cognitive impairment to normal cognitive functioning: a population-based study. PLoS One 2013;8:e59649. 
9 Flicker C, Ferris SH, Reisberg B. Mild cognitive impairment in the elderly: predictors of dementia. Neurology 1991;41:1006.

10 Petersen RC, Smith GE, Waring SC, et al. Aging, memory, and mild cognitive impairment. Int Psychogeriatr 1997;9(Suppl 1):65-9.

11 Petersen RC, Smith GE, Waring SC, et al. Mild cognitive impairment: clinical characterization and outcome. Arch Neurol 1999;56:303-8.

12 Petersen RC. Mild cognitive impairment as a diagnostic entity. $J$ Intern Med 2004;256:183-94.

13 Winblad B, Palmer K, Kivipelto M, et al. Mild cognitive impairmentbeyond controversies, Towards a consensus: report of the International Working Group on Mild Cognitive Impairment. $J$ Intern Med 2004:256:240-6.

14 Hess TM, Hinson JT. Age-related variation in the influences of aging stereotypes on memory in adulthood. Psychol Aging 2006;21:621-5.

15 Hess TM, Hinson JT, Hodges EA. Moderators of and mechanisms underlying stereotype threat effects on older adults' memory performance. Exp Aging Res 2009;35:153-77.

16 Kit KA, Tuokko HA, Mateer CA. A review of the stereotype threat literature and its application in a neurological population. Neuropsychol Rev 2008;18:132-48.

17 Steele CM. A threat in the air. How stereotypes shape intellectual identity and performance. Am Psychol 1997;52:613-29.

18 Steele CM, Aronson J. Stereotype threat and the intellectual test performance of African Americans. J Pers Soc Psychol 1995;69:797-811.

19 Barber SJ. An examination of age-based stereotype threat about cognitive decline: implications for stereotype threat research and theory development. Perspect Psychol Sci 2017;12:62-90.

20 Chasteen AL, Kang SK, Remedios JD. Aging and stereotype threat: development, process and interventions. In: Inzlicht M, Schmader T, eds. Stereotype threat theory, process, and application. Oxford University Press, 2011: 202-16.

21 Lamont RA, Swift HJ, Abrams D. A review and meta-analysis of age-based stereotype threat: negative stereotypes, not facts, do the damage. Psychol Aging 2015;30:180-93.

22 Hess TM, Auman C, Colcombe SJ, et al. The impact of stereotype threat on age differences in memory performance. $J$ Gerontol $B$ Psychol Sci Soc Sci 2003;58:P3-11.

23 Hess TM, Emery L, Queen TL. Task demands moderate stereotype threat effects on memory performance. J Gerontol B Psychol Sci Soc Sci 2009;64B:482-6.

24 Hess TM, Hinson JT, Statham JA. Explicit and implicit stereotype activation effects on memory: do age and awareness moderate the impact of priming? Psychol Aging 2004;19:495-505.

25 Levy B. Improving memory in old age through implicit selfstereotyping. J Pers Soc Psychol 1996;71:1092-107.

26 Levy BR, Zonderman AB, Slade MD, et al. Memory shaped by age stereotypes over time. J Gerontol B Psychol Sci Soc Sci 2012;67:432-6.

27 Stein R, Blanchard-Fields F, Hertzog C. The effects of age-stereotype priming on the memory performance of older adults. Exp Aging Res 2002:28:169-81.

28 Mazerolle M, Régner I, Morisset P, et al. Stereotype threat strengthens automatic recall and undermines controlled processes in older adults. Psychol Sci 2012;23:723-7.

29 Mazerolle M, Régner I, Rigalleau F, et al. Stereotype threat alters the subjective experience of memory. Exp Psychol 2015;62:395-402.

30 Chasteen AL, Bhattacharyya S, Horhota M, et al. How feelings of stereotype threat influence older adults' memory performance. Exp Aging Res 2005;31:235-60.

31 Desrichard O, Köpetz C. A threat in the elder: the impact of taskinstructions, self-efficacy and performance expectations on memory performance in the elderly. Eur J Soc Psychol 2005;35:537-52.

32 Kang SK, Chasteen AL. The moderating role of age-group identification and perceived threat on stereotype threat among older adults. Int J Aging Hum Dev 2009;69:201-20.

33 Barber SJ, Mather M, Gatz M. How stereotype threat affects healthy older adults' performance on clinical assessments of cognitive decline: the key role of regulatory fit. J Gerontol B Psychol Sci Soc Sci 2015;70:891-900.

34 Fresson M, Dardenne B, Geurten M, et al. [Formula: see text] The effect of stereotype threat on older people's clinical cognitive outcomes: investigating the moderating role of dementia worry. Clin Neuropsychol 2017;31:1306-28.

35 Haslam C, Morton TA, Haslam SA, et al. 'When the age is in, the wit is out': age-related self-categorization and deficit expectations reduce performance on clinical tests used in dementia assessment. Psychol Aging 2012;27:778-84.

36 Mazerolle M, Régner I, Barber SJ, et al. Negative aging stereotypes impair performance on brief cognitive tests used to screen for predementia. J Gerontol B Psychol Sci Soc Sci 2016;4:gbw083.
37 Folstein MF, Folstein SE, McHugh PR. 'Mini-mental state'. J Psychiatr Res 1975;12:189-98.

38 Nasreddine ZS, Phillips NA, Bédirian V, et al. The Montreal cognitive assessment, MoCA: a brief screening tool for mild cognitive impairment. J Am Geriatr Soc 2005;53:695-9.

39 Johns M, Schmader T, Martens A. Knowing is half the battle: teaching stereotype threat as a means of improving women's math performance. Psychol Sci 2005;16:175-9.

40 Chan A-W, Tetzlaff JM, Altman DG, et al. Spirit 2013 statement: defining standard protocol items for clinical trials. Ann Intern Med 2013;158:200-7.

41 Thomas Antérion C, Ribas C, Honoré-Masson S. Le questionnaire de plainte cognitive (QPC): un outil de recherche de plainte suspecte d'évoquer une maladie d'Alzheimer. In: L'Année Gérontologique. France, 2003: 17. 56-65

42 Yesavage JA, Brink TL, Rose TL, et al. Development and validation of a geriatric depression screening scale: a preliminary report. $J$ Psychiatr Res 1982;17:37-49.

43 Dubois B, Touchon J, Portet F, et al. 'Les 5 mots', épreuve simple et sensible pour le diagnostic de la maladie d'Alzheimer. Presse Méd 2002;31:1696-9.

44 Lawton MP, Brody EM. Assessment of older people: selfmaintaining and instrumental activities of daily living. Gerontologist 1969;9:179-86

45 Tabachnick BG, Fidell LS. Using multivariate statistics. 5th edn. Boston: Pearson/Allyn \& Bacon, 2007.

46 Soper D. (2006-2013). Statistics calculators version 3.0. Available: tiny.cc/sopercalc

47 Hurt CS, Burns A, Brown RG, et al. Perceptions of subjective memory complaint in older adults: the illness perception Questionnaire-Memory (IPQ-M). Int Psychogeriatr 2010;22:750-60.

48 Rust TB, Kwong See ST. Beliefs about aging and Alzheimer's disease in three domains. Can J Aging 2010;29:567-75.

49 Albert MS, DeKosky ST, Dickson D, et al. The diagnosis of mild cognitive impairment due to Alzheimer's disease: recommendations from the National Institute on Aging-Alzheimer's association workgroups on diagnostic guidelines for Alzheimer's disease. Alzheimers Dement 2011;7:270-9.

50 Alzheimer's Association. 2012 Alzheimer's disease facts and figures. Alzheimers Dement 2012;8:131-68.

51 Sarazin M, Berr C, De Rotrou J, et al. Amnestic syndrome of the medial temporal type identifies prodromal AD: a longitudinal study. Neurology 2007;69:1859-67.

52 Van der Linden M, Coyette F, Poitrenaud J. L'épreuve de rappel libre/ rappel indicé 16 items (RL/RI-16). In: Van der Linden M, Adam S, Agniel A, eds. L'évaluation des troubles de la mémoire. Présentation de quatre tests de mémoire épisodique (avec leur étalonnage). Marseille: Solal, 2004: 25-47.

53 Heart rate variability: standards of measurement, physiological interpretation and clinical use. Task force of the European Society of cardiology and the North American Society of pacing and electrophysiology. Circulation 1996;93:1043-65.

54 Lac G, Dutheil F, Brousse G, et al. Saliva DHEAS changes in patients suffering from psychopathological disorders arising from bullying at work. Brain Cogn 2012;80:277-81.

55 Dubois B, Hampel H, Feldman HH, et al. Preclinical Alzheimer's disease: definition, natural history, and diagnostic criteria. Alzheimers Dement 2016;12:292-323.

56 Daerr S, Brendel M, Zach C, et al. Evaluation of early-phase $\left[{ }^{18} \mathrm{~F}\right]-$ florbetaben PET acquisition in clinical routine cases. Neuroimage Clin 2017; $14: 77-86$.

57 Wong JT, Gallo DA. Activating aging stereotypes increases source recollection confusions in older adults: effect at encoding but not retrieval. J Gerontol B Psychol Sci Soc Sci 2019;74:633-41.

58 Sabri O, Sabbagh MN, Seibyl J, et al. Florbetaben PET imaging to detect amyloid beta plaques in Alzheimer's disease: phase 3 study. Alzheimers Dement 2015;11:964-74.

59 Seibyl J, Catafau AM, Barthel $\mathrm{H}$, et al. Impact of training method on the robustness of the visual assessment of 18 F-Florbetaben PET scans: results from a phase-3 study. J Nucl Med 2016;57:900-6.

60 Coley N, Gardette V, Cantet C, et al. How should we deal with missing data in clinical trials involving Alzheimer's disease patients? Curr Alzheimer Res 2011:8:421-33.

61 Merckelbach H, Jelicic M, Jonker C. Planting a misdiagnosis of Alzheimer's disease in a person's mind. Acta Neuropsychiatr 2012;24:60-2.

62 Herrera C, Chambon C, Michel BF, et al. Positive effects of computer-based cognitive training in adults with mild cognitive impairment. Neuropsychologia 2012;50:1871-81.

63 Reitan RM. Validity of the TRAIL making test as an indicator of organic brain damage. Percept Mot Skills 1958;8:271-6. 
64 Seo EH, Lee DY, Choo IH, et al. Normative study of the Stroop color and word test in an educationally diverse elderly population. Int $J$ Geriatr Psychiatry 2008;23:1020-7.

65 Perlstein WM, Dixit NK, Carter CS, et al. Prefrontal cortex dysfunction mediates deficits in working memory and prepotent responding in schizophrenia. Biol Psychiatry 2003;53:25-38.

66 Cardebat D, Doyon B, Puel M, et al. Évocation lexicale et sémantique chez des sujets normaux: performances et dynamiques de production en fonction du sexe, de l'âge, et du niveau d'étude[Formal and semantic lexical evocation in normal subjects. Performance and dynamics of production as a function of sex, age and educational level]. Acta Neurol Belg 1990;90:207-17.

67 Osterrieth PA. Le test de copie d'une figure complex: contribution l'étude de la perception et de la mémoire [The test of copying a complex figure: A contribution to the study of perception and memory]. Archives de Psychologie 1944;30:206-356.

68 Rey A. L'examen psychologique dans les cas d'encephalopathie traumatique [Psychological examination of traumatic encephalopathy]. Arch Psychol 1941;1941:286-340.

69 Taylor LB. Localisation of cerebral lesions by psychological testing. Neurosurgery 1969;16(CN_suppl_1):269-87.

70 Kaplan E, Goodglass H, Weintraub S. The Boston naming test. Lea \& Febiger, 1983.

71 Gonneaud J, Rauchs G, Groussard M, et al. How do we process event-based and time-based intentions in the brain? an fMRI study of prospective memory in healthy individuals. Hum Brain Mapp 2014;35:3066-82. 